\title{
Classification of Disaster Risks in the Philippines using Adaptive Boosting Algorithm with Decision Trees and Support Vector Machine as Based Estimators
}

\author{
Donata D. Acula
}

Institute of Information and Computing Sciences/ Research Center for Natural and Applied Sciences, University of Santo Tomas, Manila

* Corresponding author email: ddacula@ust.edu.ph

Received: 30 November 2020 / Revised: 31 March 2021 / Accepted: 20 May 2021 / Published: 03 June 2021

\begin{abstract}
This paper employed the intelligent approach based on machine learning categorized as base and ensemble methods in classifying the disaster risk in the Philippines. It focused on the Decision Trees, Support Vector Machine, Adaptive Boosting Algorithm with Decision Trees, and Support Vector Machine as base estimators. The research used the Exponential Regression for missing value imputation and converted the number of casualties, damaged houses, and properties into five (5) risk levels using Quantile Method. The 10-fold cross-validation was used to validate the proposed algorithms. The experiment shows that Decision Trees and Adaptive Decision Trees are the most suitable models for the disaster data with the score of more than $90 \%$, more than $75 \%$, more than $75 \%$ in all the classification metrics (accuracy, precision, recall f1-score) when applied to classification risk levels of casualties, damaged houses and damaged properties respectively.
\end{abstract}

Keywords: disaster risks, decision trees, support vector machine, adaptive boosting algorithm

\section{$1 \quad$ Introduction}

The Philippines is considered vulnerable to hazards brought by various natural calamities such as tropical cyclones, earthquakes, volcanic eruptions, and other natural disasters. This is due to the geographical location of the country in SouthEast Asia [15]. Among the stated natural disasters, tropical cyclone is one of the most destructive phenomena that brought a high number of casualties and damages in both residential and commercial infrastructures. One of the strongest tropical cyclones is the typhoon Haiyan with the local name typhoon Yolanda in 2013. It was estimated that the said typhoon brought at least 8,000 casualties with a total cost of damage of more than 2000 million USD for both agriculture and infrastructure [15], [10]. In the record of the Philippine Atmospheric, Geophysical, and Astronomical Services Administration (PAGASA), the country experiences an average of twenty (20) tropical cyclones every year [22]. Due to this natural phenomenon, the Philippine Government continues to plan to minimize the casualties and damages brought by the said calamity.

Data Science, the newest and the fastest-growing area in the field of information technology education has a bigger role and impact in the policy-making of different private and public institutions. One of the major roles of this area of study is to help the government or private institutions to develop a policy and strategic plan by applying various algorithmic models. As highlighted in the study of Kolman [11], these algorithms and algorithmic models may dictate what policy is perceived feasible. Hagen et.al [8] also concluded that visual analytics, a major area of Data Science has potentially positive impacts on policy-making practices. When Data Science was mentioned, Artificial Intelligence (AI) always comes in, especially if the type of data analysis involves predictions or classifications. Artificial Intelligence is sometimes denoted as Machine 
Learning (ML) techniques [21]. This is because ML is an application of AI that provides systems the ability to automatically learn and improve from experience without being explicitly programmed and focuses on the development of computer programs that can access data and use it to learn from themselves [20]. In this study, an ensemble algorithm is known as Adaptive Boosting (AdaBoost) Algorithm under ML Supervised Learning will be used in the classification of the level of disaster risk brought by tropical cyclones in the Philippines. Ensemble methods train multiple learners to solve the same problem and construct a set of base learners using the base learning algorithm and combine them [26]. Among the existing type of ensemble algorithms, AdaBoost was used due to its ability to identify misclassified instances that occur because of the disjunct problem or the datasets which contain instances in a class that is clustered in several separate small groups, and each group contains a small number of instances that cannot be disregarded and should be trained [12],[18]. Specifically, this study will be employing the Decision Trees (DT) and Support Vector Machine (SVM) as base learning algorithms or as base estimators. These two base estimators were used since it shows exceptional performance in adjusting the accuracy of the ensemble model [12]. After the implementation of methods, evaluation of each performance will be applied in the DT, SVM, DTAdaBoost, and DT-SVM.

The data used in this study is gathered from different government agencies such as the National Disaster Risk Reduction and Management Council (NDRRMC), Philippine Statistical Authority (PSA), Philippine Atmospheric, Geophysical, and Astronomical Services Administration (PAGASA), and Department of Health (DoH). Using the proposed method, this research will classify the level of the disaster risk in the country which serves as a dependent variable and the rest of the gathered data such as population, details regarding the tropical cyclone and the number of hospitals will be the predictors or will serve as independent variables. Historical data from 2005 to 2016 was used to verify the performance of the methods used in this study.

Aside from experimenting with a suitable machine learning algorithm for classification, this study was conducted to help the Philippines government in assessing the potential risks of the disaster. This knowledge will lead the policymakers to allocate more resources to the prone areas to reduce casualties during the phenomena.

The same study was conducted by Alquisola et al. [7] in 2018 that predicts the disaster risks using various models such as Autoregressive Integrated Moving Average (ARIMA), Discrete Wavelet Transform (DWT), and Artificial Neural Networks (ANN). The combined algorithms generated an accuracy of $68 \%$ for casualties, 39.84\% for Damaged Houses, and 33.33\% for Damaged Properties. Due to the low performance of the model, it was recommended to explore other models in predicting the risk level of tropical cyclones. This paper explored and experimented various methods to classify the risks of the typhoon in the Philippines. Using the available data coming from the government agencies, the Adaptive Boosting Algorithm, an ensemble supervised learning method was implemented. Two of the famous Based Algorithms, Decision Trees and Support Vector Machines will be used as based estimators of the Adaptive Boosting Algorithm.

\section{$2 \quad$ Related Works}

The successful implementation of AdaBoost is the key factor why this study used the said model for the classification of disaster risk. Various application fields such as medicine, business, and others proved that this model provided good performance for prediction or classification. Zarandi et al. [4] used the AdaBoost with Support Vector Regressor in modeling minimum miscibility pressure of pure/impure CO2-crude oil systems and concluded that the model gives the most acceptable and accurate result with a very satisfactory error distribution. AdaBoost was also implemented by Walker \& Jiang [23] in the demand-driven acquisition of library materials and compared it to the logistic regression model. The authors concluded that AdaBoost performs better with an accuracy of $82 \%$. In the study of Wang et al. [13] regarding the classification of the 
Donata D. Acula, J. Mod. Sim. Mater.; Vol. 4 Issue 1, pp: 7-18, 2021

large-scale traffic data, AdaBoost used to classify by automatically detecting the outliers in traffic data and the SVM used to train the model for identifying the categories of outliers. The AdaBoost-SVM achieved a classification rate of $92 \%$.

This method can also be implemented in image processing. In the study of Fan et al. [5], the group proposed the AdaBoost procedure for low-rank-based image denoising and concluded that it outperformed other denoising algorithms in terms of Peak Signal-to-Noise Ratio (PSNR) and Structural Similarity Index (SSIM). The group of Feng et al. [6], proposed this method to predict the compressive strength of concrete by employing Artificial Neural Network (ANN), Support Vector Machine, and Decision Trees as base predictors. The result of their experiment shows that the Decision Tree is the best choice for the weak learner in the boosting framework with an accuracy rate of $98 \%$. The model was also used in the case study of Liu \& Chen [13] to predict the spatial air quality index in China. It was concluded that the model is suitable for the air quality early-warning since it significantly outperforms the other models used in the experiment.

AdaBoost was also implemented in the wireless sensor networks by Yadahalli \& Nighot [16] and the result of the experiment obtained a detection rate of $98.13 \%$ with a false alarm rate of 0.38 . This was also used in the fault diagnosis and compared in the SVM. The result of the experiment conducted by Peng et al. [14] showed that AdaBoost is more efficient than SVM. He et al. [9] also applied the AdaBoost in the fault diagnosis with resampling and the simulation result achieved higher fault diagnosis accuracy than the other models.

Finally, Wu et al. [25] the model was applied in the landslide susceptibility mapping to help the decisionmakers in utilizing the land resources and achieve economic development as well as bring harmony between human beings and the fragile loess environment. The data were simulated in different models such as Alternating Decision Tree (ADT), Alternating Decision Tree with AdaBoost (ADT-AdaBoost), Alternating Decision Tree with Bagging (ADT-Bagging), and the results claimed that ADT-AdaBoost is the most efficient models among the three (3) models with a success rate of $98.4 \%$.

\section{$3 \quad$ Methodology and System Models}

The Input-Process-Output (IPO) of the research was presented in Figure 1 and the complete flow of the whole system was shown in Figure 2. The gathered data categorized as hazard variables, exposure variables, vulnerability variables, and disaster risks variables used in the study underwent preprocessing. The exponential regression was used for missing data imputation and the quantile method was implemented to classify five (5) disaster risk levels (1 -Very Low, 2-Low, 3 - Moderate, 4 - High, 5- Very High) with 0 as no risk. The range of each level per risk was presented in Table 1 . Then the data partitioning was applied where $80 \%$ of the total data used in the training of algorithms and the remaining $20 \%$ was used for testing.

After data partitioning, the data will undergo training in using the DT, SVM, DT-AdaBoost, and SVMAdaBoost. The model is then evaluated using the K-fold cross-validation with the tuning of parameters. Once validated it will be producing the accuracy of the training models. Once the parameters of the models are settled, the generated models will be used to the testing stage where the remaining $20 \%$ of the data will be fed in the same algorithms as the training stage for classification.

Finally, the metrics of the classification will be obtained such as classification matrix, accuracy, precision, recall, F1-score. The result will be used to determine if there is a significant difference in the actual and classified risk level using the non-parametric test. 


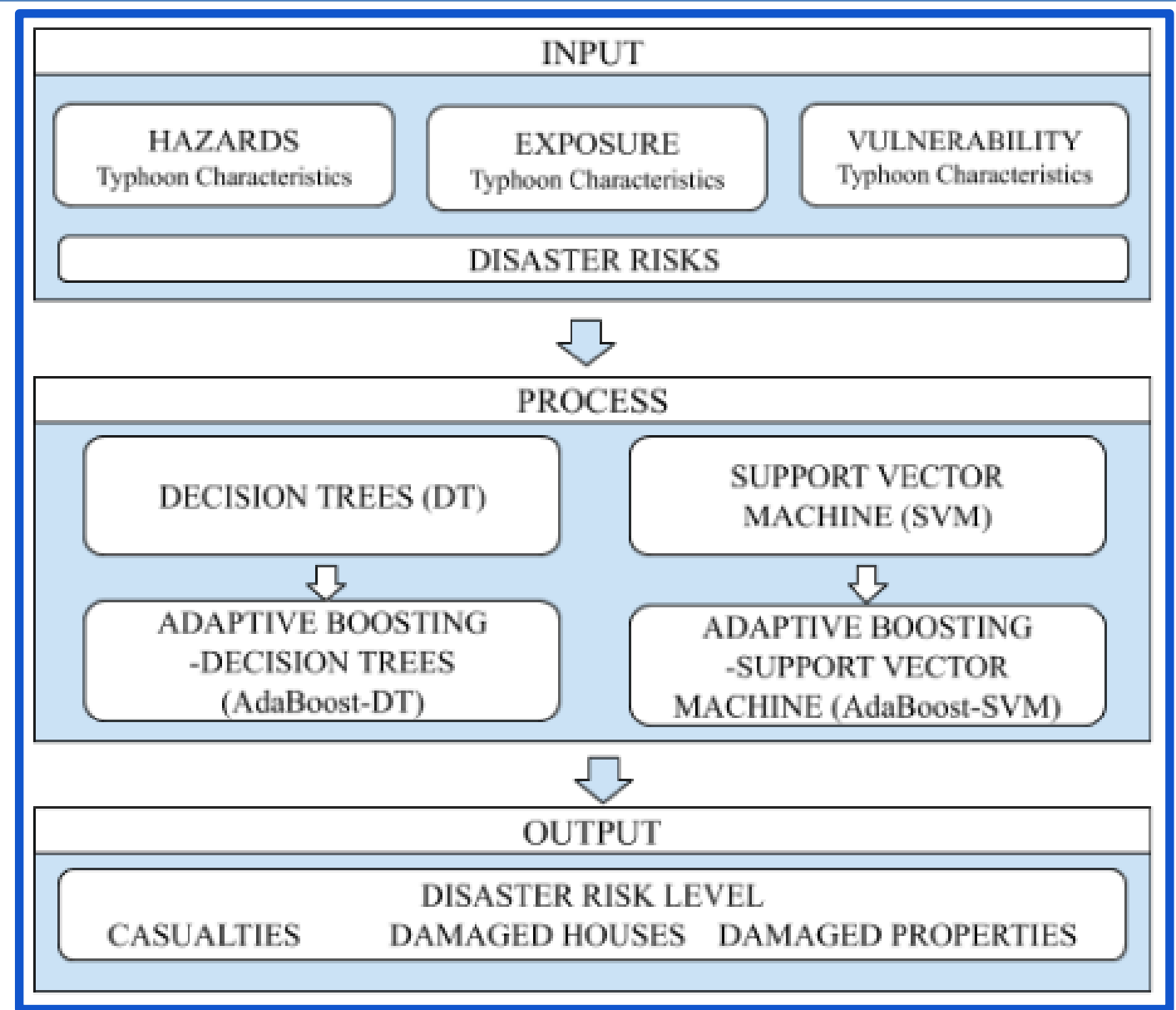

Figure 1: Conceptual Framework of the Study

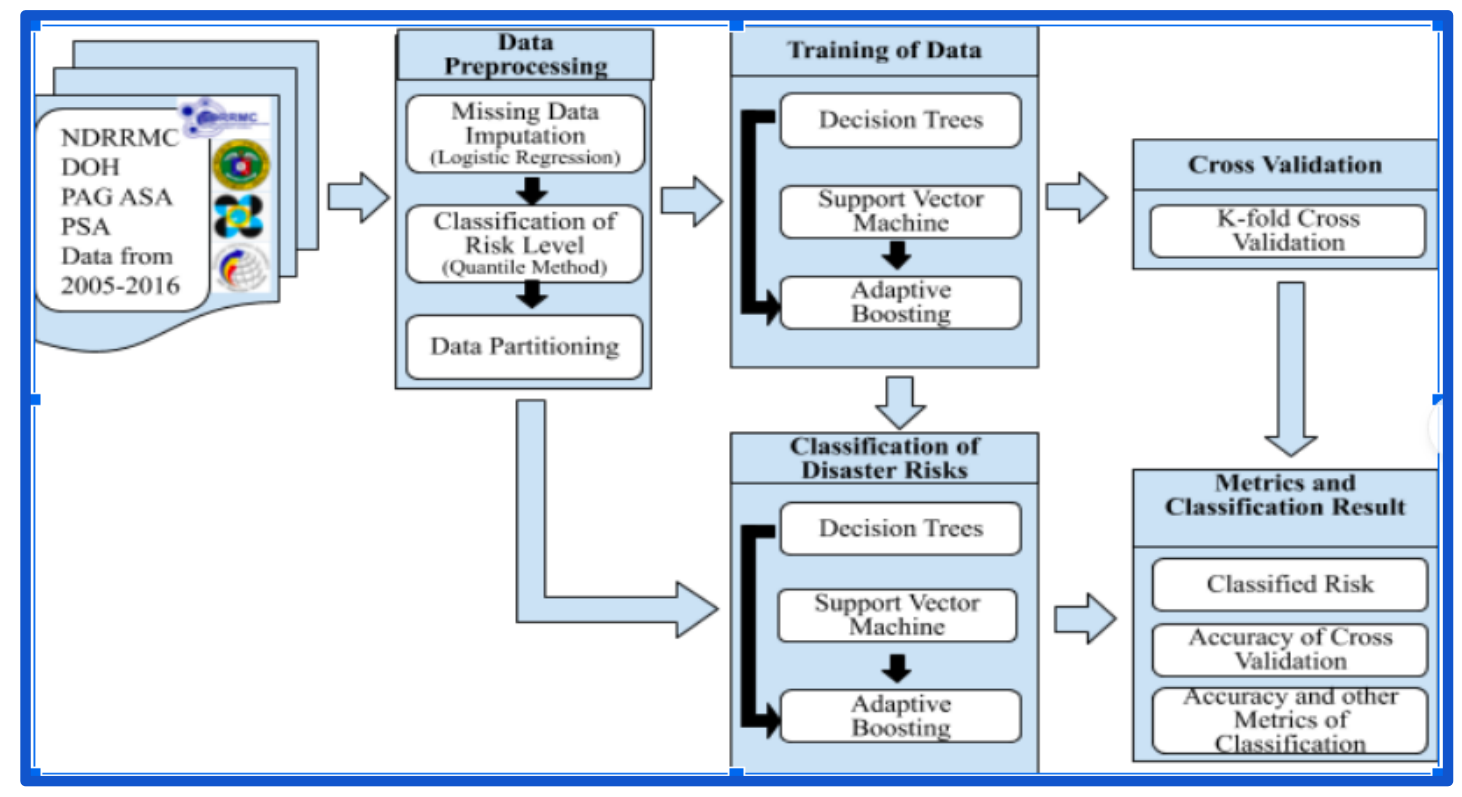

Figure 2: System Architecture of the Study 


\subsection{Exponential Regression}

The role of exponential regression in this study is to forecast the gaps of year values of the different predictors such as population. The data for the population has gaps in between years because PSA conducts surveys about population every 5 years only, sometimes every 3 years. Thus, the exponential regression was used to predict the gap year values in terms of population. The Exponential Regression model was shown in (1).

$$
f(t)=a b^{t}
$$

where:

$$
\begin{aligned}
& f(t)=\text { population after } \mathrm{t} \text { years } \\
& a=\text { initial value } \\
& b=\text { base or growth factor } \\
& t=\text { time in years }
\end{aligned}
$$

\subsection{Quantile Deviation}

Quantile Method is also known as Percentile Method is a type of data classification method where the values are distributed into groups or classes that contain an equal number of features. $t$ can also refer to dividing a probability distribution into areas of equal probability [17]. The quantile method can be used to determine the thresholds of risk level based on its use in the World Risk Index by the World Risk Report organization. The formula was shown in (2) that calculates the number of observations per class.

$$
T O C=\frac{T O}{N C}
$$

where: $\quad T O C=$ Total Observation per Class

$\mathrm{TO}=$ Total Observation

$\mathrm{NC}=$ Number of Class

The dependent variables (casualty, damaged houses, and damaged properties) in the dataset are the actual values of the risks. To be able to feed them to the classification algorithm, the quantile method was used to classify these dependent variables into five (5) classes, where one (1) has the lowest risk level and five (5) has the highest risk level. The use of the quantile classification method provided the data classes at the extremes and middles the same number of features. The representation of each class on the map is equal, and the classes are easy to compute.

\subsection{Decision Trees}

Decision trees used in this study since it does not require any prior assumptions about the probability distributions that govern the class and attributes of the data. This model also can be applied to both categorical and continuous data without requiring the attributes to be transformed into a common representation via binarization, normalization, or standardization [19]. The Pseudocode below shows the steps of Decision Tree Classifiers when used in the disaster risks data. [19]. The input will be the disaster risk data (training or testing) with instances I together with the independent and dependent variables known as attributes A. It will select the best attributes recursively and split the data and expand the nodes of the tree until the required condition is satisfied. 


\section{Decision Tree Induction}

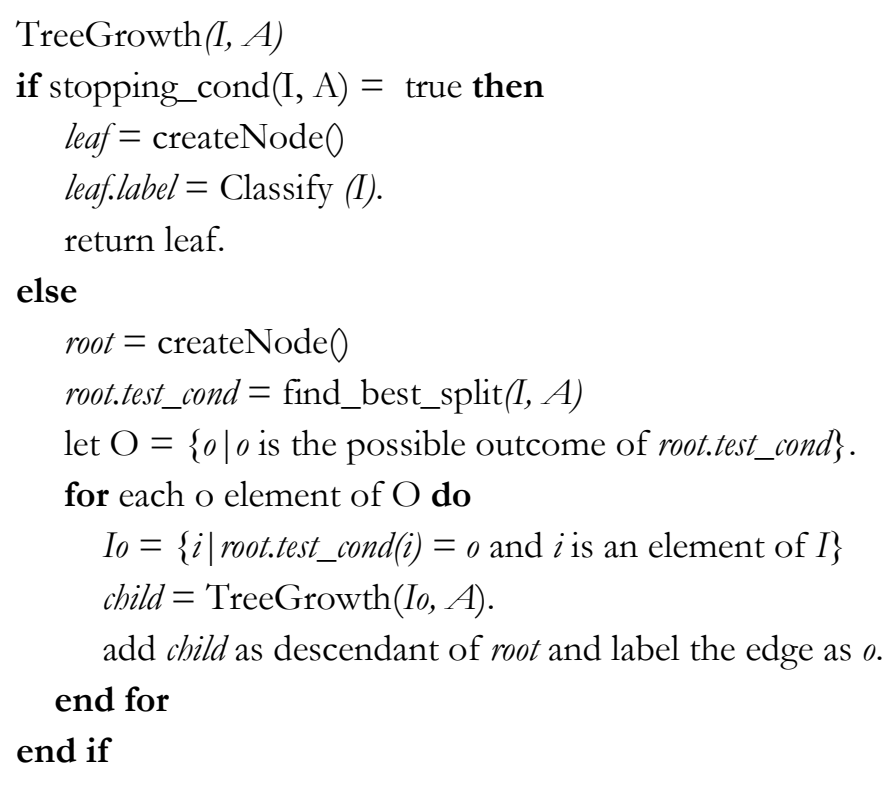

return root.

\subsection{Support Vector Machine}

Support Vector Machine is one of the widely used classification algorithms whereas the data is plotted in an $n$-dimensional space given that $n$ represents the total number of features available in the data and the value of the features lie in a particular coordinate. To perform the classification of risk level in the data, the following steps were presented in the pseudocode. The algorithm searches for the candidate support vectors represented by $\mathrm{S}$ and assumes that SV occupies a space where the parameters of the linear features of the hyper-plane are stored [23].

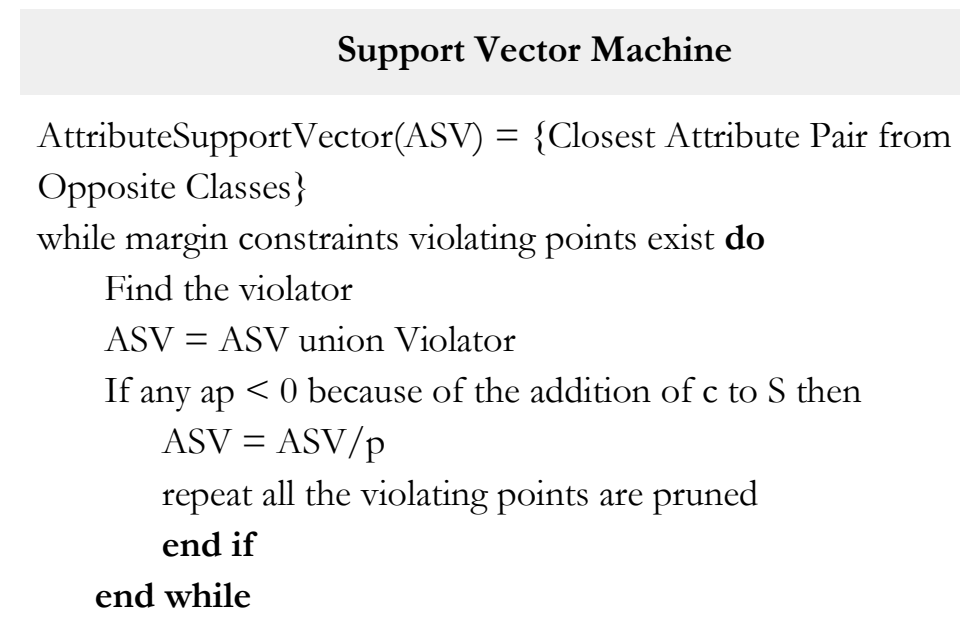

\subsection{Adaptive Boosting Algorithm}

Once the data is processed in the Decision Trees or Support Vector Machine, the model will be fed in the Adaptive Boosting Model. The procedure was presented by Chun \& Kim [3] was shown below where the data given data start with initialization of the weight which was computed as the reciprocal of the total number training data. Then it will call the weak learners from the base estimators. In this study, the weak learners are Decision Trees and Support Vector Machines. After choosing the alpha, the weights will be updated and it will produce a strong classifier. 


\section{Adaptive Boosting Algorithm}

Given: $\left(x_{1}, y_{1}\right), \ldots,\left(x_{m}, y_{m}\right) x_{i} \in X, y_{i} \in\{-1,1\}$

Initialize weight $D_{1}(i)=1 / m$

For $\mathrm{t}=1,2, \ldots, \mathrm{T}$

Call weak learn which returns weak classifier $h_{t}: X \in\{-1$,

1\}

with minimum error w.r.t. $\mathrm{D}_{\mathrm{t}}$

Choose $\alpha_{t} \in \mathrm{R}$

Update weight

$$
D_{t+1}(i)=\frac{D_{t}(i) e^{-\alpha_{t} y_{i} h_{t}\left(x_{i}\right)}}{Z_{t}}
$$

when $Z_{t}$ is a normalization factor chosen so that $D_{t+1}$ is a normal distribution

Produce the strong classifier

$$
H(x)=\operatorname{sign} \sum_{t=1}^{T} \alpha_{t} h_{t}(x)
$$

\subsection{K-Fold Cross-Validation}

$\mathrm{K}$-fold cross-validation is a procedure used to estimate the skills of the algorithms used in training the disaster risk data with $\mathrm{k}=10$. As presented by [2], it first shuffles the dataset randomly and then splits the datasets into $k$ groups. Then for every unique group, it takes the group as hold out or test data; takes the remaining groups as training data set; fits a model on the training set and evaluate it on the test set; and retain the evaluation score and discard the model. Lastly, it summarizes the skill of the model using the sample of model evaluation scores. The diagram in Figure 3 shows the $\mathrm{k}$-fold cross-validation.

\begin{tabular}{|ll|}
\hline 123 & $\mathrm{n}$ \\
\hline & \\
\hline 11765 & \\
\hline 11765 & 47 \\
\hline 11765 & 47 \\
\hline 11765 & 47 \\
\hline 11765 & 47 \\
\hline
\end{tabular}

Figure 3: K-Fold Cross-Validation

\subsection{Classification Metrics}

Classification Metrics will be used to determine the performance of different classification models presented in this paper. Confusion Matrix, Accuracy, Precision, Recall or Sensitivity, and F1 Score will be provided and computed. 


\subsubsection{Confusion Matrix}

Confusion matrix utilized for classification problems that can generate an output with two or more types of classes. It is presented in two-dimensional matrices containing actual and prediction classes whose elements were represented by True Positive (TP), True Negatives (TN), False Positives (FP), and False Negative (FN) The said matrix is presented in the table below.

Table 1: Confusion Matrix

\begin{tabular}{|c|c|c|c|}
\hline \multirow{3}{*}{ Predicted } & \multicolumn{3}{|c|}{ Actual } \\
\hline \multirow{3}{*}{} & Positives (1) & Positives (1) & Negatives (0) \\
\cline { 2 - 4 } & Negatives (0) & TP & FP \\
\cline { 2 - 4 } & & FN \\
\hline
\end{tabular}

\subsubsection{Accuracy}

Accuracy will be used to determine the correct classified risk level. The numerator will contain the correctly classified risk level while the denominator will be the total classified risk levels. Using the confusion matrix, the accuracy can be calculated using TP and TN using (4).

$$
\text { Accuracy }=\frac{T P+T N}{T P+T N+F P+F N}
$$

\subsubsection{Precision}

The precision will be used to determine the proportion of the results which are relevant and correctly classified risk levels.

$$
\text { Precision }=\frac{T P}{T P+T N}
$$

\subsubsection{Recall or Sensitivity}

Recall or sensitivity will be used to determine the proportion of True Positive and the sum of True Positive and False Positive.

$$
\text { Recall }=\frac{T P}{T P+F P}
$$

\subsubsection{F1-Score}

F1 score is used to maximize both precision and recall using the harmonic mean as shown in (7).

$$
\text { F1 }- \text { Score }=\frac{2 * \text { Precision } * \text { Recall }}{\text { Precision }+ \text { Recall }}
$$

\subsection{Paired Wilcoxon Test}

To determine if there is no difference between the actual and predicted value of the risk level, the paired Wilcoxon test was used at alpha $=5 \%$ level of significance. When the p-value is higher than the alpha the test will imply that the predicted value is the same as the actual value. Otherwise, it will lead to the conclusion that the model is not good for the prediction of disaster risk. 
Donata D. Acula, J. Mod. Sim. Mater.; Vol. 4 Issue 1, pp: 7-18, 2021

\section{$4 \quad$ Model Performance Analysis}

This section discussed the result of the models used in the experiment. The presentation was categorized as Casualties, Damaged Houses, and Damage Properties.

\subsection{Casualties}

Table 2: Comparison of Performance of Risk Level Classification for Casualties

\begin{tabular}{lcccc}
\hline & \multicolumn{3}{c}{ Classification Metrics } \\
\cline { 2 - 4 } & Accuracy & Precision & Recall & F1 Score \\
\hline DT & $92.14 \%$ & $91.60 \%$ & $92.20 \%$ & $92.00 \%$ \\
SVM & $86.04 \%$ & $74.40 \%$ & $86.20 \%$ & $79.60 \%$ \\
AdaBoostDT & $92.14 \%$ & $91.80 \%$ & $92.00 \%$ & $92.00 \%$ \\
AdaBoostSVM & $86.04 \%$ & $74.40 \%$ & $86.20 \% 0$ & $79.60 \%$ \\
DWT-ARIMA-ANN & $62.81 \%$ & $22.54 \%$ & - & -
\end{tabular}

Using the gathered data, the result for simulation of the proposed method in classifying the casualties risk level was shown in Table 2. Included in the last row of the table is the output of the previous study where Discrete Wavelet Transform-Autoregressive Integrated Moving Average - Artificial Neural Networks was implemented in the same data.

The model with the highest accuracy, precision, recall, and f1 score is Decision Trees and AdaBoost-DT with scores of more than 90\%. Support Vector Machine and AdaBoost-SVM have the same result in all the metrics. Almost a 30\% and 70\% difference was observed between the new method and the previous method in the prediction or classification of disaster risks under casualties.

\subsection{Damaged Houses}

In terms of dealing with the damaged houses, there is a very low metric score for the SVM and AdaBoostSVM with a score of less than 50\%. Whereas, DT and AdaBoost-DT achieved a good result of more than $70 \%$ in all the metrics. It was also shown that a big improvement exists as compared with the previous study using DW'T-ARIMA-ANN based on accuracy and precision.

Table 3: Performance of Risk Level Classification for Damaged Houses

\begin{tabular}{lcccc}
\hline \multirow{2}{*}{ Models } & \multicolumn{3}{c}{ Classification Metrics } \\
\cline { 2 - 5 } & Accuracy & Precision & Recall & F1 Score \\
\hline DT & $75.90 \%$ & $76.20 \%$ & $75.80 \%$ & $75.80 \%$ \\
SVM & $38.92 \%$ & $30.80 \%$ & $39.00 \%$ & $34.00 \%$ \\
AdaBoostDT & $75.56 \%$ & $76.00 \%$ & $75.60 \%$ & $75.60 \%$ \\
AdaBoostSVM & $27.81 \%$ & $15.60 \%$ & $28.00 \%$ & $14.40 \%$ \\
DWT-ARIMA-ANN & $39.84 \%$ & $31.72 \%$ & - & -
\end{tabular}

\subsection{Damaged Properties}

The result of the classification of risk level under damaged properties using the proposed models is almost similar to the result of the damaged houses' risk level. Both DT and AdaBoost-DT obtained a score of 
more than $70 \%$ for all the stated metrics while the SVM and AdaBoost-SVM garnered less than $50 \%$ for all the metrics. Table 4 also shows that the DT and AdaBoost-DT is a better method in predicting the risk level of damaged properties than the DWT-ARIMA-ANN.

To determine if there is no significant difference between the classified risk level using the proposed methods, the paired Wilcoxon test was implemented.

Table 4: Performance of Risk Level Classification for Damaged Properties

\begin{tabular}{lcccc}
\hline \multirow{2}{*}{ Models } & \multicolumn{3}{c}{ Classification Metrics } \\
\cline { 2 - 5 } & Accuracy & Precision & Recall & F1 Score \\
\hline DT & $75.04 \%$ & $75.60 \%$ & $75.00 \%$ & $75.00 \%$ \\
SVM & $36.47 \%$ & $28.40 \%$ & $36.40 \%$ & $29.40 \%$ \\
AdaBoostDT & $74.30 \%$ & $74.60 \%$ & $74.20 \%$ & $74.20 \%$ \\
AdaBoostSVM & $28.49 \%$ & $9.60 \%$ & $28.60 \%$ & $14.40 \%$ \\
DWT-ARIMA-ANN & $33.33 \%$ & $40.03 \%$ & - & -
\end{tabular}

The result of the test shows that for the three categories such as casualties, damaged houses, and damaged properties only the Decision Trees and Adaptive Boosting Decision Trees can be considered a good and acceptable model using the data. The summary of the p-value was presented in Table 5.

Table 5: P-value using Paired Wilcoxon Test

\begin{tabular}{cccc}
\hline \multirow{2}{*}{ Models } & \multicolumn{3}{c}{ p-value } \\
\cline { 2 - 4 } & Casualties & Damaged Houses & Damaged Properties \\
\hline DT & 0.89113 & 0.24869 & 0.55697 \\
SVM & $2.82022 \times 10^{-10}$ & $6.16666 \times 10^{-5}$ & 0.00047 \\
AdaBoost-DT & 0.20179 & 0.215631 & 0.19943 \\
AdaBoost-SVM & $2.82022 \times 10^{-10}$ & $7.02532 \times 10^{-5}$ & $3.729 \times 10^{-7}$
\end{tabular}

\section{$5 \quad$ Conclusions}

This research proposed a method known as ensemble algorithms under supervised learning to address the need of the Philippines government in policymaking and resource allocation during the disaster. Four methods were experimented such as Decision Trees (DT), Support Vector Machine (SVM), Adaptive Boosting Algorithm with Decision Trees as Base Estimator (AdaBoost-DT), and Adaptive Boosting Algorithm with Support Vector Machine as Base Estimator (AdaBoost-SVM). The methods were also compared in the accuracy of the previous research [7] where DWT-ARIMA-ANN was used. This research concluded that Decision Trees outperformed the remaining models. Adaptive Boosting-Decision Trees can also be considered a good model for the classification of disaster risk level due to its small difference in accuracy and other metrics when compared to Decision Trees. Also, there is no significant difference in the actual and predicted disaster risk level in the casualties, damaged houses, and damaged properties when both Decision Trees and Adaptive Boosting -Decision Trees were implemented using the Wilcoxon test. 
Donata D. Acula, J. Mod. Sim. Mater;; Vol. 4 Issue 1, pp: 7-18, 2021

Future researchers are encouraged to use the successful models in the other field of application and apply other methods to classify the risk level of the same data.

\section{Declarations}

\subsection{Competing Interests}

The author declares that no potential conflict of interest exists related to this article.

\subsection{Acknowledgments}

The author would like to thank the different government agencies in the Philippines (NDRRMC, PSA, DOH, PAGASA) for sharing the data which was used in this study and the University of Santo Tomas for funding this research. The trust and encouragement from Dr. Maribel G. Nonato (Vice-Rector for Research and Innovation), Dr. Bernard John V. Tongol ( RCNAS Director), and Jerralyn T. Padua (IICS Director) was greatly appreciated.

\section{How to Cite this Article:}

D. D. Acula, "Classification of Disaster Risks in the Philippines using Adaptive Boosting Algorithm with Decision Trees and Support Vector Machine as Based Estimators", J. Mod. Sim. Mater., vol. 4, no. 1, pp. 7-18, Jun. 2021.

\section{References}

[1] Alloghani, M., Aljaaf, A., Hussain, A. et al. Implementation of machine learning algorithms to create diabetic patient readmission profiles. BMC Med Inform Decis Mak 19, 253 (2019). https://doi.org/10.1186/s12911-019-0990-x

[2] Brownlee, J. (2019, August 8). A Gentle Introduction to $k$-fold Cross-Validation. Machine Learning Mastery. https://machinelearningmastery.com/k-fold-cross-validation/

[3] Chun, J., Kim, W. 3D face pose estimation by a robust real-time tracking of facial features. Multimed Tools Appl 75, 15693-15708 (2016). https://doi.org/10.1007/s11042-014-2356-9

[4] Dargahi-Zarandi, A., Hemmati-Sarapardeh, A., Shateri, M., Menad, N. A., \& Ahmadi, M. (2020). Modeling minimum miscibility pressure of pure/impure $\mathrm{CO} 2$-crude oil systems using adaptive boosting support vector regression: Application to gas injection processes. Journal of Petroleum Science and Engineering, 184, 106499. https://doi.org/10.1016/j.petrol.2019.106499

[5] Fan, L., Li, X., Fan, H., \& Zhang, C. (2019). An adaptive boosting procedure for low-rank based image denoising. Signal Processing, 164, 110-124. https://doi.org/10.1016/j.sigpro.2019.06.004

[6] Feng, D.-C., Liu, Z.-T., Wang, X.-D., Chen, Y., Chang, J.-Q., Wei, D.-F., \& Jiang, Z.-M. (2020). Machine learning-based compressive strength prediction for concrete: An adaptive boosting approach. Construction and Building Materials, 230, 117000. https://doi.org/10.1016/j.conbuildmat.2019.117000

[7] G. L. V. Alquisola, D. J. A. Coronel, B. M. F. Reolope, J. N. A. Roque and D. D. Acula, "Prediction and Visualization of the Disaster Risks in the Philippines Using Discrete Wavelet Transform (DWT), Autoregressive Integrated Moving Average (ARIMA), and Artificial Neural Network (ANN)," 2018 3rd International Conference on Computer and Communication Systems (ICCCS), Nagoya, 2018, pp. 146-149, https://doi.org/10.1109/CCOMS.2018.8463238.

[8] Hagen, L., Keller, T. E., Yerden, X., \& Luna-Reyes, L. F. (2019). Open data visualizations and analytics as tools for policy-making. Government Information Quarterly, 36(4), 101387. https://doi.org/10.1016/j.giq.2019.06.004

[9] He, Y.-L., Zhao, Y., Hu, X., Yan, X.-N., Zhu, Q.-X., \& Xu, Y. (2020). Fault diagnosis using novel AdaBoost based discriminant locality preserving projection with resamples. Engineering Applications of Artificial Intelligence, 91, 103631. https://doi.org/10.1016/j.engappai.2020.103631

[10] Hernandez, J. Y. (2015, July 31). Damage Caused by Typhoon Haiyan in the Philippines, Review of Structural Regulations and Practice, and Research Developments in Wind Engineering. JAWE. https://www.jstage.jst.go.jp/article/jawe/40/3/40_270/_article

[11] Kolkman, D. (2020). The usefulness of algorithmic models in policy making. Government Information Quarterly, 37(3), 101488. https://doi.org/10.1016/j.giq.2020.101488

[12] Lee, W., Jun, C.-H., \& Lee, J.-S. (2017). Instance categorization by support vector machines to adjust weights in AdaBoost for imbalanced data classification. Information Sciences, 381, 92-103. https://doi.org/10.1016/j.ins.2016.11.014

[13] Liu, H., \& Chen, C. (2020). Spatial air quality index prediction model based on decomposition, adaptive boosting, and three-stage feature selection: A case study in China. Journal of Cleaner Production, 265, 121777. https://doi.org/10.1016/j.jclepro.2020.121777

[14] P. Peng, Y. Zhang, Y. Wu and H. Zhang, "An Effective Fault Diagnosis Approach Based On Gentle AdaBoost and AdaBoost.MH," 2018 IEEE International Conference on Automation, Electronics and Electrical Engineering (AUTEEE), Shenyang, China, 2018, pp. 812, https://doi.org/10.1109/AUTEEE.2018.8720764.

[15] Philippines: Disaster Management Reference Handbook (March 2018) - Philippines. (2018, March 30). ReliefWeb. https://webcache.googleusercontent.com/search?q=cache:pvzngTylj1sJ:https://reliefweb.int/report/philippines/philippines-disastermanagement-reference-handbook-march-2018+\&cd=1\&hl=en\&ct=clnk\&gl=ph 
[16] S. Yadahalli and M. K. Nighot, "Adaboost based parameterized methods for wireless sensor networks," 2017 International Conference On Smart Technologies For Smart Nation (SmartTechCon), Bangalore, 2017, pp. 1370-1374, https://doi.org/10.1109/SmartTechCon.2017.8358590

[17] Stephanie, B. (2019, August 26). Quantile: Definition and How to Find Them in Easy Steps. Statistics How To. https://www.statisticshowto.com/quantile-definition-find-easy-steps/

[18] Taherkhani, A., Cosma, G., \& McGinnity, T. M. (2020). AdaBoost-CNN: An adaptive boosting algorithm for convolutional neural networks to classify multi-class imbalanced datasets using transfer learning. Neurocomputing, 404, 351-366. https://doi.org/10.1016/j.neucom.2020.03.064

[19] Tan, P. N., Steinbach, M., Karpatne, A., \& Kumar, V. (2019). Introduction to Data Mining. Pearson.

[20] Team, E. S. (2020, May 29). What is Machine Learning? A definition. Expert System. https://expertsystem.com/machine-learningdefinition/\#:\%7E:text=Machine\%20learning\%20is\%20an\%20application,use\%20it\%20learn\%20for\%20themselves

[21] Toh, T. S., Dondelinger, F., \& Wang, D. (2019). Looking beyond the hype: Applied AI and machine learning in translational medicine. EBioMedicine, 47, 607-615. https://doi.org/10.1016/j.ebiom.2019.08.027

[22] Tropical Cyclone Information (n.d.). Retrieved from http://bagong.pagasa.dost.gov.ph/climate/tropical-cyclone-information

[23] Walker, K. W., \& Jiang, Z. (2019). Application of adaptive boosting (AdaBoost) in demand-driven acquisition (DDA) prediction: A machine-learning approach. The Journal of Academic Librarianship, 45(3), 203-212. https://doi.org/10.1016/j.acalib.2019.02.013

[24] Wang, L.-L., Ngan, H. Y. T., \& Yung, N. H. C. (2018). Automatic incident classification for large-scale traffic data by adaptive boosting SVM. Information Sciences, 467, 59-73. https://doi.org/10.1016/j.ins.2018.07.044

[25] Wu, Y., Ke, Y., Chen, Z., Liang, S., Zhao, H., \& Hong, H. (2020). Application of alternating decision tree with AdaBoost and bagging ensembles for landslide susceptibility mapping. CATENA, 187, 104396. https://doi.org/10.1016/j.catena.2019.104396

[26] Zhou, Z. (2012). Ensemble Methods: Foundations and Algorithms (Chapman \& Hall/Crc Machine Learning \& Pattern Recognition) (1st ed.). Chapman and Hall/CRC.

Publish your research article in AIJR journals-

$\checkmark \quad$ Online Submission and Tracking

$\checkmark \quad$ Peer-Reviewed

$\checkmark \quad$ Rapid decision

$\checkmark \quad$ Immediate Publication after acceptance

$\checkmark \quad$ Articles freely available online

$\checkmark \quad$ Retain full copyright of your article.

Submit your article at journals.aiji.org
Publish your books with AIJR publisher-

$\checkmark \quad$ Publish with ISBN and DOI.

$\checkmark \quad$ Publish Thesis/Dissertation as a Book.

$\checkmark \quad$ Publish Monograph.

$\checkmark \quad$ Publish Edited Volume/ Book.

$\checkmark \quad$ Publish Conference Proceedings

$\checkmark \quad$ Retain full copyright of your books.

Submit your manuscript at books.aijr.org 